Universitas, Volumen 1, Año 1, 2007,39-46

(C) 2007 UNAN-León, Editorial Universitaria

\title{
Perfil epidemiológico de la caries dental y enfermedad periodontal, en Nicaragua, año 2005.
}

\author{
Dr. Carlo Medina ${ }^{1}$, Jorge Antonio Cerrato ${ }^{2 *}$, Miriam del Socorro Herrera ${ }^{3 *}$ \\ ${ }^{3}$ Departamento de Cirugía Oral, Facultad de Odontología, Universidad Nacional Autónoma de Nicaragua, León. \\ (UNAN-León). \\ ${ }^{2}$ Departamento de Odontología Preventiva y Social, Facultad de Odontología Universidad Nacional Autónoma de \\ Nicaragua, León. (UNAN-León). \\ ${ }^{1}$ Centro de Investigaciones en Sistemas de Salud, Instituto Nacional de Salud Pública, Cuernavaca, Morelos, \\ México.
}

\section{RESUMEN}

La caries y enfermedad periodontal, son problemas de salud pública por sus efectos: dolor, deterioro funcional, disminución de calidad de vida, causa de problemas sistémicos como obstrucción de vías aéreas, corazón, cerebro, propensión a neumonía, problemas gastrointestinales, piel, sistema inmunológico, síndrome de fatiga crónica, poniendo en riesgo la vida de personas con válvulas cardíacas previamente dañadas, o causar nacimientos prematuros, razón por la que se consideró de vital importancia determinar el perfil epidemiológico de estas enfermedades en Nicaragua. Se realizó estudio descriptivo, transversal, muestra de 1800 personas, edades 5, 12, 15, 35-44, 65-74 años, urbano y rural, ambos sexos, tomando criterios que establece la OMS "Investigación de Salud Oral, Métodos Básicos"[9], sobre estudios epidemiológicos de caries dental y enfermedad periodontal: 12 puntos muestrales. Resultados: Prevalencia ataque de caries $90.61 \%$. Prevalencia de enfermedad periodontal, de $88.23 \%$. Conclusiones: Población nicaragüense con altos índices de caries y enfermedad periodontal, no existiendo diferencia de prevalencia de caries, respecto al sexo, pero aumenta a medida que aumenta la edad, siendo mayor en la zona rural. La prevalencia de la enfermedad periodontal, es mayor en mujeres, aumenta a medida que aumenta la edad, siendo mayor en la zona rural.

Palabras claves: Perfil epidemiológico, Caries dental, Enfermedad periodontal.

\section{INTRODUCCIÓN}

Cuando se tabulan las causas de extracciones dentarias, basándose en la masa de la población, la caries dental aparece como responsable de aproximadamente un 40 a $45 \%$ del total, pudiendo atribuirse otro 40 a $45 \%$ a la enfermedad periodontal y el resto a una variedad de razones. En tal sentido dos son las causas más comunes e importantes de la pérdida de los dientes: caries dental y enfermedad periodontal, siendo su factor local predisponente la placa dentobacteriana. ${ }^{[1]}$.

La caries dental y la enfermedad periodontal se presentan tan prevalentes y severas en las poblaciones, que son consideradas los principales problemas de salud pública en el campo de la odontología.

La caries dental y la enfermedad periodontal están relacionadas a factores generales tales como: económicos, educacionales y sociales. Se ha visto que a más bajo nivel de educación, a mayor deficiencia de higiene oral y más bajo nivel económico mayor es la incidencia de caries dental y enfermedad periodontal. ${ }^{[2]}$

\footnotetext{
*Autores para correspondencia: jacerrato@unanleon.edu.ni, miriams33@hotmail.com
}

La caries dental es una enfermedad crónica y la más frecuente en la dentición humana. Una vez que se presenta, sus manifestaciones persisten a lo largo de toda la vida, incluso cuando las lesiones han sido tratadas $^{[3]}$.

Las periodontopatías, son alteraciones patológicas de cualquier origen, que atacan los tejidos periodontales; ellas comprenden las diversas enfermedades gingivales y las manifestaciones periodontales de otras enfermedades. ${ }^{[1]}$

En salud pública, como principio general, los recursos son siempre menores que los problemas, por lo tanto una de sus metas es el uso de metodologías que precisen el aumento de la productividad, menor tiempo y bajo costo.

Siendo la caries dental y la enfermedad periodontal, problemas de salud pública y por lo tanto, las causas más comunes e importantes de la pérdida de dientes y afección de tejidos blandos y duros dentro de la cavidad oral, se consideró que es de vital importancia determinar el perfil epidemiológico de estas enfermedades en los habitantes de Nicaragua, año 2005, en aras de obtener un conocimiento actualizado y objetivo sobre dicho tema, orientar acciones de intervención. 
En un estudio epidemiológico de salud bucal en niños de $6,7,8,12$ y 15 años, de escuelas públicas de Nicaragua, realizado1997, realizado por Narváez Pineda Alejandra, se refleja que la prevalencia de caries dental en niños de 12 y 15 años, fue de 79 y $88 \%$ respectivamente, que el CPOD en los niños de 12 años, fue de 2.8 y en los de 15 años fue de 4.5 .

Nicaragua, es un país en vías de desarrollo y en el área de la odontología, posee una relativa escasez de información respecto a indicadores epidemiológicos oficiales. Si se logra mejorar el estado actual del conocimiento en el área de la salud bucal, entonces será posible desarrollar estrategias que aborden los problemas de salud bucal, lo cual repercutirá en mejorar la salud general de la población.

Dadas las limitaciones económicas actuales del país, es necesario priorizar los recursos destinados a programas de salud bucal, para asegurar su orientación a aquellas medidas que logren un impacto con la mejor relación costo-beneficio.

Es importante el hecho de que para realizar este estudio, se utilizó la encuesta que internacionalmente recomienda la Organización Mundial de la Salud, OMS, lo que servirá para poder hacer comparaciones con los datos estadísticos de otros países de la región, tener una visión global del problema y así poder orientar a las autoridades competentes, a la toma de políticas de intervención adecuadas, en función de disminuir la prevalencia de estas enfermedades bucales en nuestro medio. ${ }^{[4]}$

\section{DISEÑO METODOLOGICO}

\subsection{Tipo de Estudio:}

Descriptivo de corte transversal.

\subsection{Universo de Estudio:}

Está constituido por el conjunto de todas las personas que habitan los Municipios de Chinandega, Managua, Masaya, Río San Juan, Matagalpa, Ocotal, RAAS (Puerto Cabezas), con edades de 5 años, 12 años, 15 años, de 35 a 44 años y de 65 a 74 años, en las zonas urbanas, y rurales, tanto del sexo femenino como masculino.

\subsection{Muestra: se obtuvo una muestra por conglomerados:}

Se tomaron como referencia los criterios que establece el manual "Investigación de Salud Oral, Métodos Básicos" de la OMS, sobre estudios epidemiológicos de caries dental y enfermedad periodontal en grupos
Total de personas examinadas en cada punto muestral: 150 personas.

Total de la muestra: 150 personas $X 12$ puntos muestrales: 1800 personas.

\begin{tabular}{|c|c|c|c|}
\hline \multirow{2}{*}{$\begin{array}{c}\text { Edades } \\
\text { (En Años) }\end{array}$} & \multicolumn{3}{|c|}{ Area Urbana } \\
\hline & $\begin{array}{c}\text { Total de } \\
\text { Individuos }\end{array}$ & Varones & Mujeres \\
\hline 5 & 240 & 120 & 120 \\
\hline 12 & 240 & 120 & 120 \\
\hline 15 & 240 & 120 & 120 \\
\hline 35 a 44 & 240 & 120 & 120 \\
\hline 65 a 74 & 240 & 120 & 120 \\
\hline Totales & 1200 & 600 & 600 \\
\hline
\end{tabular}

\begin{tabular}{|l|l|l|l|}
\hline \multirow{2}{*}{$\begin{array}{c}\text { Edades } \\
\text { (en Años) }\end{array}$} & \multicolumn{3}{|c|}{ Area Rural } \\
\cline { 2 - 4 } & $\begin{array}{c}\text { Total de } \\
\text { Individuos }\end{array}$ & Varones & Mujeres \\
\hline 5 & 120 & 60 & 60 \\
\hline 12 & 120 & 60 & 60 \\
\hline 15 & 120 & 60 & 60 \\
\hline 35 a 44 & 120 & 60 & 60 \\
\hline 65 a 74 & 120 & 60 & 60 \\
\hline Totales & 600 & 300 & 300 \\
\hline
\end{tabular}

poblacionales, el cual indica que para enfermedades muy prevalentes como caries dental y enfermedad periodontal.

Se obtuvieron los listados de los grados de esos centros escolares que tenían en niños y niñas con las edades índices, (5, 12 y 15 años).

Mediante el sistema de muestreo aleatorio simple, se obtuvieron al azar, 30 nombres de los diferentes grupos de edades índices (5, 12 y 15 años) de cada punto muestral de las diferentes zonas geográficas, siendo 15 personas del sexo masculino y 15 del sexo femenino.

Las unidades de análisis con las edades de 35 a 44 años y con las edades de 65 a 74 se obtuvieron de los pobladores de las juntas receptoras de votos seleccionadas, visitando las casas de las manzanas o zonas, explicándoles los objetivos del estudio y solicitándoles su cooperación. Se preguntó si había personas en esos rangos de edades, lo cual se comprobaba con la cédula, hasta completar 15 varones y 15 mujeres en cada punto muestral.

Las personas que se negaron a ser estudiadas, se sustituyeron con otras personas que llenaban los criterios de inclusión. 


\subsection{Unidad de observación:}

Son los dientes y las encías de cada persona seleccionada en la muestra.

\subsection{Unidad de análisis:}

Cada una de las personas seleccionadas en la muestra.

\subsection{Instrumento de recolección de datos:}

Se elaboró una ficha para el registro de la información individual de cada unidad de análisis, en base a los objetivos de la investigación y con los criterios de los Índices CPOD, ceod y CPITN.

\subsection{Método de recolección de la información:}

Previo a la recolección de la información, el equipo investigador, compuesto por dos Odontólogos docentes de la Facultad de Odontología UNAN LEON, un Docente de la Centro de Investigaciones en Sistemas de Salud, Instituto Nacional de Salud Publica, Cuernavaca, Morelos, México y un equipo auxiliar de veintitrés egresados de Odontología, hizo una estandarización de criterios de los índices de caries dental CPOD: (dientes permanentes cariados, perdidos y obturados), ceo (dientes temporales cariados, extracción indicada y obturados) y de enfermedad periodontal CPITN (índice periodontal de la comunidad), utilizando para la estandarización la prueba de Kappa, igual a un 85\% para los índices CPOD y ceo y de un $65 \%$ para el índice CPITN.

Una vez seleccionadas las unidades de análisis, se les realizó el examen visual- clínico, sentando al paciente cómodamente en una silla, auxiliados con un espejo bucal plano y luz natural comenzando con el examen dental, utilizando los criterios del Índice CPOD, ceo, (según la edad del paciente), comenzando por la parte más posterior de la hemiarcada superior derecha, hasta llegar a la parte más posterior de la hemiarcada superior izquierda, seguidamente se examinó desde la parte mas posterior de la hemiarcada inferior izquierda, hasta concluir en la parte más posterior de la hemiarcada inferior derecha.

En los dientes que no se apreciaba muy bien si había o no lesión cariosa, se secó la superficie dental, con una pera de aire.

En un formato específico diseñado para el estudio, que contiene un odontograma y los sextantes a examinar periodontalmente, se asentaron los resultados de las variables clínicas en relación a caries dental, anotando los datos obtenidos. Seguidamente, se realizó el examen de las encías, utilizando el índice periodontal CPITN, siguiendo el mismo orden del examen dental.

\section{RESULTADOS Y DISCUSIÓN}

La dentición temporal presentó una prevalencia de caries de 76,7 por 100 niños de 5 años y solamente un 23,3 por cada 100 niños no presentó la enfermedad; 74,2 niños y niñas del área urbana de 5 años por cada 100 examinados, han sido atacados por la caries dental; 85 niños del área rural por cada 100 examinados, han sido atacados por la caries dental; 78,3 niñas del área rural, por cada 100 examinadas, han sido atacadas por la caries dental, lo cual evidencia que el ataque de la caries dental inicia a edades muy tempranas, trayendo como consecuencia problemas funcionales, psicológicos, nutricionales y posteriormente problemas de mal oclusión.

La población urbana masculino presentó una media de tres dientes cariados y con una desviación típica de tres, la población femenina presentó una media de tres dientes cariados con una desviación típica de tres, quedando un ceo de tres y una desviación típica de tres; la población rural masculino presentó una media de cinco dientes cariados, desviación típica cuatro; femenino, media de cuatros dientes cariados, desviación típica de tres, en estos se presentó un ceo de cinco con una desviación típica de cuatro en el masculino y en el femenino una media de cuatro con una desviación típica de cuatro. Ver Tabla 1.

En la dentición permanente el 94,1 de personas > de 12 años por cada cien, presentó caries dental, solamente un 5,9 por cada cien no fueron atacados por esta patología. 92,1 hombres > de 12 años del área urbana por cada cien han sido atacados por la caries dental; 94, 2 mujeres > de 12 años del área urbana por cada cien han sido atacadas por esta misma patología; 95 hombres > de 12 años del área rural por cada cien han sido atacados por la caries dental; 97,1 mujeres $>$ de 12 años del área rural por cada cien han sido atacadas por la caries dental. Ver Tabla 2. 
Tabla 1. PROMEDIO DE DIENTES ATACADOS POR CARIES EN DENTICION TEMPORAL, EN NICARAGUA, AÑO 2005.

\begin{tabular}{|c|c|c|c|c|c|c|c|c|}
\hline \multicolumn{3}{|c|}{ DIENTES TEMPORALES } & \multicolumn{6}{|c|}{ SEXO } \\
\hline \multirow{10}{*}{$\begin{array}{c}\text { Localización } \\
\text { Geográfica }\end{array}$} & & & \multicolumn{3}{|c|}{ Masculino } & \multicolumn{3}{|c|}{ Femenino } \\
\hline & \multirow{4}{*}{ Urbano } & & Recuento & Media & $\begin{array}{l}\text { Desviación } \\
\text { Típica }\end{array}$ & Recuento & Media & $\begin{array}{l}\text { Desviación } \\
\text { Típica }\end{array}$ \\
\hline & & Cariados & 120 & 3 & 3 & 120 & 3 & 3 \\
\hline & & $\begin{array}{l}\text { Extracciones } \\
\text { Indicadas }\end{array}$ & 120 & 0 & 0 & 120 & 0 & 1 \\
\hline & & Obturados & 120 & 0 & 1 & 120 & 0 & 1 \\
\hline & & $\mathrm{Ceo}$ & 120 & 3 & 3 & 120 & 3 & 3 \\
\hline & & Cariados & 60 & 5 & 4 & 60 & 4 & 3 \\
\hline & Purol & $\begin{array}{l}\text { Extracciones } \\
\text { Indicadas }\end{array}$ & 60 & 0 & 1 & 60 & 0 & 1 \\
\hline & Nural & Obturados & 60 & 0 & 0 & 60 & 0 & 1 \\
\hline & & $\mathrm{Ceo}$ & 60 & 5 & 4 & 60 & 4 & 4 \\
\hline
\end{tabular}

Tabla 2. PREVALENCIA DE CARIES EN DIENTES PERMANENTES SEGÚN LOCALIZACION GEOGRAFICA Y SEXO, EN NICARAGUA, AÑO 2005.

\begin{tabular}{|c|c|c|c|c|c|c|c|}
\hline & & & & \multicolumn{4}{|c|}{ CARIES DIENTES PERMANENTES } \\
\hline & & & & \multicolumn{2}{|c|}{ SIN CARIES } & \multicolumn{2}{|c|}{ CON CARIES } \\
\hline \multirow{6}{*}{$\begin{array}{c}\text { Localización } \\
\text { geográfica }\end{array}$} & & & & Recuento & $\%$ & Recuento & Prevalencia $\times 100$ \\
\hline & \multirow{2}{*}{ urbano } & \multirow{2}{*}{ SEXO } & MASCULINO & 38 & 7,9 & 442 & 92,1 \\
\hline & & & FEMENINO & 28 & 5,8 & 452 & 94,2 \\
\hline & & & & & & & \\
\hline & \multirow{2}{*}{ rural } & \multirow{2}{*}{ SEXO } & MASCULINO & 17 & 5,0 & 228 & 95,0 \\
\hline & & & FEMENINO & 2 & 2,9 & 233 & 97,1 \\
\hline
\end{tabular}

Respecto a la prevalencia de caries según la edad y área geográfica, se observó que en los hombres de la zona urbana, el 81.7 hombres de 12 años han sido atacados por la caries dental. A los 15 años el $88,3 \%$ han sido atacados por caries dental, de los 34-45 años en este mismo sexo el $100 \%$ presentó ataque de caries y de 64-75 años el 98,3\% presento la enfermedad.

En las mujeres de la zona urbana, el $83,3 \%$ de las de 12 años presentó ataque de caries dentales, el $94,2 \%$ de las de 15 años presentó esta patología; en las mujeres con edades de $34-45$, el $99,2 \%$ presentó el ataque de caries y de los $64-75$ años, el 100\% presentó la enfermedad.
En la zona rural, los jóvenes de 12 años el 86,7 \% presentó ataque de caries, los de 15 años el 95\% padece la enfermedad, los de 34 a 45 años, el 98.3\% han sido atacados por caries y los de 64 a 75 años, el $100 \%$ fueron afectados. Las niñas de 12 años de la zona rural el $93,3 \%$ sufre este padecimiento y las niñas de 15 años el 96,7\% la padece. Las mujeres de 34-45 años el $100 \%$ sufre la enfermedad y las de 64-75 años el $98,3 \%$ también la padece. Ver Tabla 3. 
Tabla 3. PREVALENCIA DE CARIES EN DIENTES PERMANENTES SEGÚN LOCALIZACION GEOGRAFICA, SEXO Y EDAD, EN NICARAGUA, AÑO 2005.

\begin{tabular}{|c|c|c|c|c|c|c|}
\hline \multirow[t]{3}{*}{$\begin{array}{l}\text { LOCALIZACION } \\
\text { GEOGRAFICA }\end{array}$} & \multirow[t]{3}{*}{ SEXO } & \multirow[t]{3}{*}{$E D A D$} & \multicolumn{4}{|c|}{ CARIES EN DIENTES PERMANENTES } \\
\hline & & & \multicolumn{2}{|c|}{ SIN CARIES } & \multicolumn{2}{|c|}{ CON CARIES } \\
\hline & & & $n$ & $\%$ & $n$ & $\%$ \\
\hline \multirow{9}{*}{ URBANO } & \multirow{4}{*}{ MASCULINO } & 12 AÑOS & 22 & 18,3 & 98 & 81,7 \\
\hline & & $15 A \tilde{N} O S$ & 14 & 11,7 & 106 & 88,3 \\
\hline & & 34-45 AÑOS & 0 & 0,0 & 120 & 100,0 \\
\hline & & $64-75$ AÑOS & 2 & 1,7 & 118 & 98,3 \\
\hline & & & & & & \\
\hline & \multirow{4}{*}{ FEMENINO } & 12 AÑOS & 20 & 16,7 & 100 & 83,3 \\
\hline & & 15 AÑOS & 7 & 5,8 & 113 & 94,2 \\
\hline & & 34-45 AÑOS & 1 & 0,8 & 119 & 99,2 \\
\hline & & $64-75$ AÑOS & 0 & 0,0 & 120 & 100,0 \\
\hline & & & & & & \\
\hline \multirow{9}{*}{ RURAL } & \multirow{4}{*}{ MASCULINO } & 12 AÑOS & 8 & 13,3 & 52 & 86,7 \\
\hline & & 15 AÑOS & 3 & 5,0 & 57 & 95,0 \\
\hline & & 34-45 AÑOS & 1 & 1,7 & 59 & 98,3 \\
\hline & & $64-75$ AÑOS & 0 & 0,0 & 60 & 100,0 \\
\hline & & & & & & \\
\hline & \multirow{4}{*}{ FEMENINO } & 12 AÑOS & 4 & 6,7 & 56 & 93,3 \\
\hline & & 15 AÑOS & 2 & 3,3 & 58 & 96,7 \\
\hline & & 34-45 AÑOS & 0 & 0,0 & 60 & 100,0 \\
\hline & & $64-75$ AÑOS & 1 & 1,7 & 59 & 98,3 \\
\hline
\end{tabular}

El promedio nacional CPOD, es de 10.25 y a los 12 años es de 4, a los 15 años es de 6; de 34-45 años es de 13 y de 64-75 años es de 18, en las mujeres el CPOD es de 11 y en los hombres de 10.

La prevalencia de enfermedad periodontal (sangrado, tártaro, bolsa < de $5 \mathrm{~mm}$, bolsas > de $5 \mathrm{~mm}$ ) presentó el siguiente comportamiento: en varones del área urbana de 12 años fue de $76,7 \%$, los de 15 años presentaron una prevalencia de $67,5 \%$; los de $34-45$ años la prevalencia fue de $91,7 \%$ y los de $64-75$ años presentaron una prevalencia de $95 \%$.

En el área rural el 98,4 \% de los varones de 12 años presenta la enfermedad, en los de 15 años está presente en el $95 \%$, en los de $34-45$ años la prevalencia fue de $96.7 \%$ y en los de $64-75$ años presentaron una prevalencia de $100 \%$.

En mujeres del área urbana de 12 años, el $77.5 \%$ presenta la enfermedad; en las de 15 años está presente en un $72.5 \%$; en las de $34-45$ años la prevalencia de enfermedad periodontal es de $90.8 \%$ y en las de $64-75$ años la prevalencia es de $95.8 \%$.

En los varones del área rural, los de 12 años presentaron una prevalencia de enfermedad periodontal de $98.3 \%$, los de 15 años presentaron una prevalencia de 95\%; los de $34-45$ años la prevalencia fue de $96.7 \%$ y los de 64-75 años presentaron una prevalencia de $100 \%$.

En mujeres del área rural, en las de 12 años, hay un 95\% de prevalencia de enfermedad periodontal, en las de 15 años está presente en un 100\%; al igual que las de 34-45 años y en las de 64-75 años la prevalencia es de $98.3 \%$.

Al grupo de mujeres y varones se aplicó la prueba Chicuadrado de Pearson dando un resultado de 10,827; con 4 grados de libertad y una significancia estadística de 0,029 resultando significativo en el nivel 0,05. Ver Tabla 4. 
Tabla 4. PREVALENCIA DE ENFERMEDAD PERIODONTAL SEGÚN LOCALIZACION GEOGRAFICA, SEXO Y EDAD

\begin{tabular}{|c|c|c|c|c|c|c|c|}
\hline \multicolumn{8}{|c|}{ ENFERMEDAD PERIODONTAL } \\
\hline \multirow{10}{*}{ URBANO } & \multirow{5}{*}{ MASCULINO } & & $\begin{array}{c}\% \\
\text { SANOS }\end{array}$ & $\begin{array}{c}\% \\
\text { SANGRADOS }\end{array}$ & $\begin{array}{c}\% \\
\text { TARTAROS }\end{array}$ & $\begin{array}{c}\% \text { BOLSAS } \leq \\
D E 5 \mathrm{~mm}\end{array}$ & $\begin{array}{c}\% B O L S A S> \\
D E 5 \mathrm{~mm}\end{array}$ \\
\hline & & 12 AÑOS & 23,3 & 21,7 & 55,0 & 0,0 & 0,0 \\
\hline & & 15 AÑOS & 32,5 & 18,3 & 49,2 & 0,0 & 0,0 \\
\hline & & 34-45 AÑOS & 8,3 & 5,0 & 47,5 & 27,5 & 11,7 \\
\hline & & $64-75$ AÑOS & 5,0 & 5,0 & 30,8 & 0,0 & 34,2 \\
\hline & & & & & & & \\
\hline & \multirow{4}{*}{ FEMENINO } & 12 AÑOS & 22,5 & 27,5 & 50,0 & 0,0 & 0,0 \\
\hline & & 15 AÑOS & 27,5 & 25,8 & 46,7 & 24,2 & 0,0 \\
\hline & & 34-45 AÑOS & 9,2 & 16,7 & 45,8 & 22,5 & 4,2 \\
\hline & & $64-75$ AÑOS & 4,2 & 6,7 & 45,0 & 0,0 & 21,7 \\
\hline & & & & & & & \\
\hline \multirow{9}{*}{ RURAL } & \multirow{4}{*}{ MASCULINO } & 12 AÑOS & 1,7 & 6,7 & 91,7 & 0,0 & 0,0 \\
\hline & & 15 AÑOS & 5,0 & 31,7 & 63,3 & 0,0 & 0,0 \\
\hline & & 34-45 AÑOS & 3,3 & $0,0 \%$ & 30,0 & 35,0 & 31,7 \\
\hline & & $64-75$ AÑOS & 0,0 & 1,7 & 6,7 & 43,3 & 48,3 \\
\hline & & & & & & & \\
\hline & \multirow{4}{*}{ FEMENINO } & 12 AÑOS & 5,0 & 15,0 & 80,0 & 0,0 & 0,0 \\
\hline & & 15 AÑOS & 0,0 & 18,3 & 81,7 & 0,0 & 0,0 \\
\hline & & 34-45 AÑOS & 0,0 & 3,3 & 41,7 & 33,3 & 21,7 \\
\hline & & $64-75$ AÑOS & 1,7 & 0,0 & 11,7 & 35,0 & 51,7 \\
\hline
\end{tabular}

A estas localizaciones geográficas se aplicó la prueba de Chi- cuadrado de Pearson dando un resultado de 102, 486 con 4 grados de libertad y una significancia estadística de 0.000 en el nivel 0.05 .

En un estudio epidemiológico de salud bucal en niños de $6,7,8,12$ y 15 años, de escuelas públicas de Nicaragua, realizado en 1997, por Narváez Pineda Alejandra, se refleja que la prevalencia de caries dental en niños de 12 y 15 años, fue de 79 y $88 \%$ respectivamente, que el CPOD en los niños de 12 años, fue de 2.8 y en los de 115 años fue de 4.5 .

Es sorprendente ver que a medida que se avanza en el tiempo y hablando de metas propuestas para el año 2000 por la OMS, en este estudio se presente una prevalencia de caries dental de $90.6 \%(\mathrm{~N}=1631)$, un ceo promedio de 4.5 y un CPOD promedio de 10.25 , lo cual es clasificado por la misma entidad como muy severo. Estos datos nos pueden indicar que los planes y programas preventivos a nivel nacional, no están siendo optimizados.
En otros países como México se observa también comportamientos diferentes, en ese país se observó que en el estado de Oaxaca, el $78 \%$ de los niños de 12 años de edad están afectados; San Luis Potosí, presenta un $83 \%$ de Prevalencia de caries y en Tamaulipas una prevalencia de $82 \%$. Algo muy relevante encontrado en la república mexicana, fue que en el Distrito Federal el $97 \%$ de los niños de 12 años de edad, presentó al menos una lesión cariosa en la boca. En Estados Unidos de Norte América, en 1988-1991, la prevalencia fue de $45 \%$ en dentición permanente en niños y adolescentes de 5 a 17 años de edad y los de 5 a 11 años de edad, presentaron una prevalencia de $26 \%$. ${ }^{[13]}$

En Costa Rica, en el último estudio nacional de 1992, en la población de 12 años, se encontró un índice CPOD, de 4,9. los valores máximos y mínimos fueron los de Limón con un CPOD de 4,0 y Punta Arena de 6,0. ${ }^{[8]}$

En El Salvador, el $82 \%$ de la población en general, padece de caries dental. ${ }^{[11]}$ 
En Cuba, según los resultados de la encuesta de salud bucal realizada en 1995, el 43,6 de los niños de 5 y 6 años se encontraban libres de caries dental, mientras que en niños de 12 años el índice CPOD, es de 1, 86, superior a la meta propuesta para el año 2000. ${ }^{[8]}$ Esto podría ser posible debido a la distribución equitativa, a la optimización de los recursos y a la ejecución correcta de los planes y programas que sobre salud bucal implementa el sistema de salud cubano.

En cuanto a la variable localización geográfica se nota como el comportamiento de la caries dental y la enfermedad periodontal, es muy variable en los diferentes países, esto podría deberse a que algunos sectores tienen menor educación en salud oral lo que implementa la aparición de caries dental y enfermedad periodontal, reflejándose que en nuestro caso, existe una diferencia significativa.

Respecto a la enfermedad periodontal, un estudio (2279 personas), en niños entre 8 y 9 años y entre 12 y 13 años de edad de zonas urbanas y rurales de Argentina, presentaron que el predominio y lo severo de la gingivitis incrementa con la edad, solamente $2.7 \%$ y de los de 7 años de edad presentaron gingivitis severa, mientras que los de 14 años presentaron un $27.2 \%$. El número de segmentos involucrados también incrementó de 0.0 a 0.6 respectivamente. ${ }^{[11]}$

Los grupos intermedios entre 8 y 12 años, mostraron valores intensos los cuales soportaron estas afirmaciones.

La gingivitis fue también estudiada en una muestra al azar de 700 niños mexicanos entre las edades de 11 y 17 años, los resultados mostraron un valor significativo del Índice Gingival de 1.26 indicando predominio similar a los estudios brasileños descritos previamente. Una diferencia significante estadísticamente entre muchachos y muchachas fueron reportados..$^{[9]}$

Maltz, valoró las condiciones orales en estudiantes (de 8 a 10 años) en Porto Alegre, Brasil sobre el tiempo, aunque un $70 \%$ se declinó en caries predominando sobre los 21 años que fue reportado, virtualmente ningún mejoramiento de las condiciones gingivales ocurrió sobre el mismo período. ${ }^{[8]}$

La presente evolución confirma la creencia que la gingivitis está ampliamente dispersa, con una tendencia mínima hacia el predominio más alto en grupos socioeconómicos bajos y finalmente más severos en hombres que en mujeres. El estudio por Pinto, que comprendió un número grande de individuos representativos de las áreas urbanas brasileños mostraron figuras comparables a otras partes del mundo, en las que se permitieron cálculos de un predominio total de enfermedades severas, de un rango de 4 a $19 \%$ de personas afectadas.

En algunos estudios al azar de más de 10.000 personas en Brasil, había la tendencia de estar más afectados severamente, el sector socio-económico menos favorecido, lo cual se reflejaba en todos los grupos de edad. En el grupo de edad media (35 y 44 años de edad) el predominio del sector con enfermedades severas, fue en la capa socio-económico más bajo, con $5.5 \%$ mientras que la más alta fue de $4.5 \% .{ }^{[8]}$

\section{CONCLUSIONES}

El Perfil Epidemiológico de la caries dental y la enfermedad periodontal en la población de Nicaragua, indica la alta prevalencia de estas enfermedades, manifestando las siguientes características:

El promedio del índice ceo indica que no hay diferencia en cuanto al sexo, pero en cuanto a la ubicación geográfica es mayor el promedio del índice ceo, en el área rural.

El promedio general del índice CPOD es de 10.25, siendo a los 12 años el CPOD de 4, aumenta conforme la edad y en cuanto al sexo no hay mayor diferencia.

La prevalencia del ataque de caries dental, inicia a edades muy tempranas y aumenta a medida que aumenta la edad, al igual que la prevalencia de la enfermedad periodontal, aumenta a medida que aumenta la edad.

No existe diferencia de la prevalencia de caries dental respecto al sexo, pero si en la prevalencia de la enfermedad periodontal, siendo mayor en el sexo femenino.

Existe diferencia de la prevalencia de caries dental y enfermedad periodontal respecto a la zona geográfica, siendo mas afectada la población rural.

\section{AGRADECIMIENTOS}

- ASDI / SAREC, por el apoyo económico, para realizar el trabajo de campo de este estudio, a través del programa "Pequeñas Ayudas para la Investigación" de la Vice-Rectoría de Investigación y Post Grado, UNAN-LEÓN.

- Al Consejo Supremo Electoral, SILAIS de los Departamentos involucrados, y MEDCD.

- Al Dr. Joel Esquivel Muñoz. Por estandarizar criterios del índice CPITN, al equipo de apoyo.

- A los estudiantes del V curso de Odontología año 2005, que formaron el equipo de apoyo.

- A Dirigentes Comunales y Padres de Familias. 
- Especialmente a: niños, adolescentes, jóvenes y adultos que fueron parte de la muestra, e hicieron posible la realización del presente estudio.

\section{BIBLIOGRAFÍA}

1. Katz, S. y col., (1993), "Odontología preventiva en acción", 3a. edición, Editorial Médica panamericana, México.

2. Chávez, M., (1962), Odontología Sanitaria, $2 a$. edición.

3. Shafer, W., (1987), Tratado de Patología Bucal, editorial FL, 4a. edición, Nueva Editorial interamericana, México.

4. Organización Mundial de la Salud, (1990), Investigación de Salud Oral, Métodos Básicos, Primera Edición, editorial, Trillas, México.

5. Carranza F., (1998), Periodontología Clínica de Glickman, 7ma. Edición, Nueva editorial Interamericana, Mcgraw- Hill, México.

6. Newbrun E, (1991), Cariologia. Editorial Limusa, Mexico, p. 1- 26

7. Herrera Miriam, (2002), "Caries dental e indicadores de riesgo en escolares de León, Nicaragua. Tesis de maestría, Universidad Autónoma de CampecheMéxico.

8. Kaste LM et al., (1996): Coronal caries in the primary and permanent Dentition Of. children and adolescent 1-17 years of age; United States (1988-1991). J Dent Res.

9. Boletín Epidemiológico, (1984), Secretaria de Salud, México.

10. Modelo de atención en salud oral en los sistemas sanitarios de El Salvador, (1999), OPS - OMS.

11. La Salud en las América, volumen 2, edición (1998), Organización Panamericana de la Salud. Publicación Científica $N^{\circ} 569$.

12. Corrales y Morales, (1999), Prevalencia de caries dental en estudiantes de 12-14 años de edad, del Instituto Nacional de Occidente "Benito Mauricio Lacayo", León-Nicaragua. Tesis de licenciatura

13. Espinoza G. y cols., (1999) Prevalencia de caries dental en escolares de 12, 18 años de edad del municipio de Quezalguaque, León- Nicaragua. Tesis de licenciatura.
14. Narváez A. y cols., (1997): Estudio epidemiológico de salud bucal en niños de 6, 7, 8,12 y 15 años de escuelas y colegios públicos en Nicaragua.

15. Genco RJ, Goldman HM, (1993), Periodoncia: Edit. linteramericana Mc Graw Hill, 1 Edición, México.

16. The American Academy of Periodontology. Periodontal diagnosis and diagnostic aids in: Proceedings of the Word Whorkshop in Clinical Periodontics. AAP (1989).

17. The Dental Clinics of North America. Periodontics: W:B. Sauders Company, (1988): 32

18. Lindhe J (1986): Periodontogía Clínica; Edit Panamericana, 1a. edición, México.

19. Pérez Z., (1997), Prevalencia de placa de bacteriana y caries dental en niños escolares de escuelas publicas y privadas de la ciudad de Diriamba, Nicaragua. Tesis de licenciatura.

20. Índice Periodontal en la comunidad y sus necesidades de tratamiento (CPITN), Facultad de Odontología.

21. Ramos Hernández Elisheva, (2006), ensayo,"El dentista puede salvar tu vida"; Selecciones de Agosto, 61-67. 